Revista de Matemática: Teoría y Aplicaciones 2003 10(1-2) : 77-90

CIMPA - UCR - CCSS ISSN: 1409-2433

\title{
LOS VALORES DEL JUEGO DE PARADA ÓPTIMA PARA MEDIAS ARITMÉTICAS DE VARIABLES DE BERNOULLI
}

\author{
Jaime Lobo* ${ }^{*}$ Santiago Cambronero**
}

Recibido: 19 Feb 2002

\begin{abstract}
Resumen
Estudiamos problemas de parada óptima para promedios generalizados de variables de Bernoulli idénticamente distribuidas, tomando los valores en el conjunto $D=\left\{d_{0}, d_{1}\right\}$. Obtenemos una fórmula de recurrencia en el caso de horizonte finito, la cual da el valor del juego en términos de problemas asociados de horizonte menor, así como el tiempo de parada óptima en estos casos. Además, presentamos una serie de aplicaciones al estudio de propiedades del valor como función de estos parámetros.
\end{abstract}

Palabras clave: Problemas de parada óptima, promedios generalizados, descomposición de tiempos de parada, clase suficiente de tiempos de parada, fórmulas de recurrencia, variables de Bernoulli.

\begin{abstract}
We study optimal stopping problems for generalized averages of identically distributed Bernoulli variables, taking values in the set $D=\left\{d_{0}, d_{1}\right\}$. We obtain a recurrent formula in the finite horizon case, which gives the value of the game in terms of associated problems of smaller horizon. This allows us to create algorithms for computing the value of the game, as well as the optimal stopping time in these cases. Moreover, we present a series of aplicattions to the study of properties of the value as a function of the parameters.
\end{abstract}

Keywords: Stopping times problems, generalized means, stopping times decomposition, sufficient class of stopping times, recurrence formulas, Bernoulli variables.

\section{Mathematics Subject Classification: 60G40.}

\footnotetext{
${ }^{*}$ CIMPA, Escuela de Matemática, Universidad de Costa Rica, 2060 San José, Costa Rica. E-Mail: jlobo@cariari.ucr.ac.cr

${ }^{* *}$ Misma dirección que J. Lobo. E-Mail: scambro@emate.ucr.ac.cr
} 


\section{Introducción}

En un espacio $(\Omega, A, P)$ se da una sucesión $X_{1}, \ldots, X_{n}, \ldots$ de variables aleatorias independientes de Bernoulli. Esto es, la ley $G$ es no degenerada y está concentrada en un conjunto $D=\left\{d_{0}, d_{1}\right\}$, con $d_{0}<d_{1}$. Se tiene entonces $G\left(\left\{d_{0}\right\}\right)=1-p, G\left(\left\{d_{1}\right\}\right)=p$, donde $0<p<1$. Se define la filtración $\mathcal{F}=\left(\mathcal{F}_{n}\right)_{n \in \mathbb{N}}$ generada por la sucesión de variables: $\mathcal{F}_{n}=\sigma\left(X_{1}, \ldots, X_{n}\right)$, no completada por $P$. Esto significa que cada $\mathcal{F}_{n}$ es una tribu finita cuyos elementos son de la forma $\left\{\omega:\left(X_{1}, . ., X_{n}\right) \in B\right\}$, con $B \subseteq D^{n}$. Denotamos por

$$
S_{n}=X_{1}+\cdots+X_{n}
$$

Por $\mathcal{T}^{N}$ se denota el conjunto de tiempos de parada $T$ de $\mathcal{F}$, tales que $T \leq N$, y por $\mathcal{T}$ el conjunto de tiempos de parada finitos de $\mathcal{F}$.

En el problema que tratamos se consideran procesos de ganancias como promedios generalizados de las $S_{n}$, de la forma

$$
W_{n}=\frac{a+S_{n}}{m+n}
$$

definidos por los parámetros $a \in \mathbb{R}, m \in \mathbb{N}$. Para cada entero $N \geq 1$ estudiamos los valores:

$$
V_{N}(a, m)=\sup _{T \in \mathcal{T}^{N}} E\left(W_{T}\right), \quad V(a, m)=\sup _{T \in \mathcal{T}} E\left(W_{T}\right)
$$

es decir, los valores de los juegos de parada óptima de horizonte $N$ y horizonte infinito, respectivamente.

En el caso $a=m=0$, el problema es conocido como el de las sumas ponderadas (estudiadas por ejemplo en [1], [2], [3]). En el caso presente de promedios generalizados, el proceso $W$ es markoviano pero no homogéneo, lo que impide obviamente aplicar en forma directa la teoría de parada óptima para procesos markovianos homogéneos (expuesta por ejemplo en el capítulo 5 de [1]), en la que se dan efectivamente fórmulas recurrentes para generar $\operatorname{los} V_{N}$.

En nuestro trabajo ofrecemos un tipo de fórmula recurrente para la sucesión $\left(V_{N}\right)_{N \geq 1}$, deduciendo de ella una para $V$. Es necesario para este efecto establecer primero algunos resultados técnicos sobre descomposición de tiempos de parada de $\mathcal{F}$, así como la existencia de una clase suficiente de tiempos de parada para los juegos de este tipo. Se ofrecen además diferentes aplicaciones de estas fórmulas recurrentes, tanto al estudio teórico de los valores como al cálculo de los mismos.

Supondremos en adelante que $\Omega=D^{\mathbb{N}}, P=\bigotimes_{i=1}^{\infty} G$, siendo $X_{n}$ la función $n$-ésima coordenada en $\Omega$.

\section{Preliminares}

Se considera el operador de traslación (shift) de coordenadas en el espacio canónico $\Omega$, definido por $\theta\left(x_{1}, x_{2}, \ldots\right)=\left(x_{2}, x_{3}, \ldots\right)$. Una propiedad elemental de este operador es la 
siguiente:

$$
\left(\theta^{k}\right)^{-1}\left(\mathcal{F}_{n}\right) \subseteq \mathcal{F}_{n+k}
$$

en el sentido de imágenes inversas de conjuntos. Los siguientes lemas introducen los conceptos de collage y de splitting de tiempos de parada.

Lema 2.1 (Operación collage) Sea $\left(A_{\lambda}\right)_{\lambda \in L}$ una partición de $\Omega$ por eventos de $\mathcal{F}_{k}$. Supongamos dada una familia de variables aletorias $\left(R_{\lambda}\right)_{\lambda \in L}$, tal que $R_{\lambda}$ es la constante 0 o bien un tiempo de parada finito de $\mathcal{F}$. Entonces la variable $T$ definida en cada $A_{\lambda}$ por $: T=k+R_{\lambda} \circ \theta^{k}$, es un tiempo de parada mayor o igual que $k$.

Para una demostración, ver [5].

Lema 2.2 (Operación splitting) Sea $T$ un tiempo de parada de $\mathcal{F}, T \geq k$. Entonces en cada átomo de $\{T>k\}$ existe un único tiempo de parada $T^{\prime}$, relativo a $\mathcal{F}$, tal que $T=k+T^{\prime} \circ \theta^{k}$ en ese átomo. Si $T \in \mathcal{T}^{N}$, entonces $T^{\prime} \in \mathcal{T}^{N-k}$.

Una versión general de este resultado existe, debido a Courrière-Priouret (ver [2], o para un caso análogo al presente, $[6$, cap2]).

Definición 2.1 Se define la clase $\mathcal{T}^{N}(\mathcal{K})$ por:

$$
\mathcal{T}^{N}(\mathcal{K})=\left\{T \in \mathcal{T}^{N}:\{T>1\}=\left\{X_{1} \in \mathcal{K}\right\}\right\}
$$

Aplicamos los resultados anteriores al caso $k=1$. Sea $\mathcal{K} \subseteq D$, y $T \in \mathcal{T}^{N}(\mathcal{K})$. Sea $j=\operatorname{card}(\mathcal{K})$, y $\rho$ una biyección de $\mathcal{K}$ en $\{1, \ldots, j\}$. Según el lema 2.2 , para cada $d \in \mathcal{K}$ existe un tiempo de parada en $\mathcal{T}^{N-1}$ que representa a $T$ en el átomo $\left\{X_{1}=d\right\}$, y que denotamos por $T_{\rho(d)}$. Definimos ahora la aplicación:

$$
\begin{aligned}
\varphi_{\mathcal{K}}^{N}: \mathcal{T}^{N}(\mathcal{K}) & \rightarrow \bigotimes_{i=1}^{j} \mathcal{T}^{N-1} \\
T & \mapsto\left(T_{1}, \ldots, T_{j}\right) .
\end{aligned}
$$

El siguiente resultado se demuestra en [5].

Teorema 1 La aplicación anterior es biyectiva.

Definición 2.2 Consideramos la siguiente clase de tiempos de parada:

$$
\mathcal{C}^{N}=\left\{T \in \mathcal{T}^{N}: \text { existen reales } c_{1}, \ldots, c_{N} \text { tales que } T=\inf \left\{n: S_{n} \geq c_{n}\right\}\right\} .
$$

Definición 2.3 Si $\mathcal{A}$ es subconjunto de $\mathcal{T}^{N}$, decimos que $\mathcal{A}$ es suficiente para el problema de parada óptima de proceso $W$, si se cumple

$$
V_{N}=\sup _{T \in \mathcal{A}} E\left(W_{T}\right)
$$


El siguiente teorema pone de manifiesto la relevancia de la clase $\mathcal{C}^{N}$. Definimos

$$
f_{N}(n, x)=V_{N-n}(a+x, m+n), \quad f(n, x)=V(a+x, m+n) .
$$

Usaremos además la notación

$$
\bar{V}_{N}(a, m)=V_{N}(a, m)-\frac{a}{m}(\text { para } m \neq 0), \quad \bar{V}_{N}(a, 0)=V_{N}(a, 0),
$$

y similarmente en el caso de horizonte infinito. El siguiente lema será de gran utilidad.

Lema 2.3 Para cada $m$ y $N$ enteros fijos, las funciones $V_{m}, V_{m}^{N}$ son convexas y crecientes, estrictamente en el caso de $V_{m}^{N}$. Si $m>0$ las funciones $\bar{V}_{m}, \bar{V}_{m}^{N}$ son convexas y estrictamente decrecientes.

Prueba: Es fácil comprobar que $a \rightarrow W_{T}(a, m)$ es estrictamente creciente para todo tiempo $T$, obteniendo el crecimiento de $V_{m}(\cdot)$, y el crecimiento estricto de $V_{m}^{N}(\cdot)$. La convexidad es consecuencia del hecho que los valores del juego son supremos de funciones afines (ver [7], por ejemplo). Finalmente, el crecimiento estricto de $\bar{V}_{m}^{N}$ y $\bar{V}_{m}$ se obtiene de la desigualdad:

$$
E\left(\frac{a+\varepsilon+S_{T}}{m+T}\right)-\frac{a+\varepsilon}{m} \leq E\left(\frac{a+S_{T}}{m+T}\right)-\frac{a}{m}-\frac{\varepsilon}{m(m+1)} .
$$

Lema 2.4 Para cada $N \geq 1$ y $m \geq 1$, los conjuntos $\left\{a \in \mathbb{R}: \bar{V}_{N}(a, m) \leq 0\right\}$ y $\{a \in \mathbb{R}: \bar{V}(a, m) \leq 0\}$ son intervalos no vacíos de la forma $\left[c,+\infty\left[\right.\right.$, y contienen a $m d_{1}$.

Prueba: El caso de horizonte finito es evidente, dado que $\bar{V}_{m}^{N}(a)$ es el máximo de una familia finita de funciones afines con pendiente negativa. En el caso de $\bar{V}_{m}(a)$ basta observar que para $a \geq m d_{1}$ y cualquier tiempo $T \in \mathcal{T}^{N}$ se tiene:

$$
\frac{a+S_{T}}{m+T}-\frac{a}{m}=\frac{m S_{T}-a T}{(m+T) m} \leq \frac{\left(m d_{1}-a\right) T}{m(m+T)} \leq 0 .
$$

En [5] demostramos el siguiente teorema.

Teorema 2 La clase $\mathcal{C}^{N}$ es una clase suficiente de tiempos de parada para el proceso $W$. El tiempo de parada $T_{N}^{*} \in \mathcal{C}^{N}$ definido por

$$
T_{N}^{*}=\inf \left\{n \leq N: f_{N}\left(n, S_{n}\right) \leq \frac{a+S_{n}}{m+n}\right\},
$$

es óptimo. Además se tiene:

$$
\left\{x: f_{N}(n, x) \leq \frac{a+x}{m+n}\right\}=\left\{x: \bar{V}_{N-n}(a+x, m+n) \leq 0\right\}=\left[c_{n},+\infty[,\right.
$$

donde los $c_{n}=c_{n}^{N}(a, m)$ definen $a T_{N}^{*}$. 


\section{Fórmulas de recurrencia para $V$ y $V_{N}$}

\subsection{Caso de horizonte finito}

Definiendo $\mu=E\left(X_{1}\right)=(1-p) d_{0}+p d_{1}$, es evidente de la definición que

$$
V_{1}(a, m)=\frac{a+\mu}{m+1}
$$

Se define

$$
\begin{aligned}
& \mathcal{T}_{0}^{N}=\mathcal{T}^{N}\left(\left\{d_{0}\right\}\right)=\left\{T \in \mathcal{T}^{N}:\{T>1\}=\left\{X_{1}=d_{0}\right\}\right\}, \\
& \mathcal{T}_{1}^{N}=\mathcal{T}^{N}\left(\left\{d_{0}, d_{1}\right\}\right)=\left\{T \in \mathcal{C}^{N}: T>1\right\}
\end{aligned}
$$

y luego $\mathcal{C}_{i}^{N}=\mathcal{C}^{N} \cap \mathcal{T}_{i}^{N}$, para $i=0,1$. Nótese que $\mathcal{C}^{N}$ es la unión disjunta:

$$
\mathcal{C}^{N}=\{1\} \cup \mathcal{C}_{0}^{N} \cup \mathcal{C}_{1}^{N}
$$

y por lo tanto, en virtud del teorema 2 tenemos

$$
\begin{aligned}
V_{N} & =\operatorname{máx}\left(E\left(W_{1}\right), \sup _{T \in \mathcal{C}_{0}^{N}} E\left(W_{T}\right), \sup _{T \in \mathcal{C}_{1}^{N}} E\left(W_{T}\right)\right) \\
& =\operatorname{máx}\left(E\left(W_{1}\right), \sup _{T \in \mathcal{T}_{0}^{N}} E\left(W_{T}\right), \sup _{T \in \mathcal{T}_{1}^{N}} E\left(W_{T}\right)\right),
\end{aligned}
$$

un hecho que será crucial en la demostración del teorema siguiente.

Teorema 3 (Fórmula de recurrencia para $V_{N}$ ) Para $N>1$ se tiene

$$
V_{N}(a, m)=\frac{a+\mu}{m+1}+\left[(1-p) \bar{V}_{N-1}\left(a+d_{0}, m+1\right)+p \bar{V}_{N-1}\left(a+d_{1}, m+1\right)^{+}\right]^{+} .
$$

Equivalentemente:

$$
V_{N}(a, m)=\frac{a+\mu}{m+1}+E\left(\bar{V}_{N-1}\left(a+X_{1}, m+1\right)^{+}\right) .
$$

Prueba: Para $T \in \mathcal{T}_{0}^{N}$ se puede escribir

$$
\begin{aligned}
E\left(W_{T}\right) & =E\left(\left(\frac{a+X_{1}}{m+1}\right) \mathbf{1}_{\left\{X_{1}=d_{1}\right\}}+\left(\frac{a+S_{T}}{m+T}\right) \mathbf{1}_{\left\{X_{1}=d_{0}\right\}}\right) \\
& =\frac{a+\mu}{m+1}+E\left(\frac{a+S_{T}}{m+T}-\frac{a+d_{0}}{m+1}\right) \mathbf{1}_{\left\{X_{1}=d_{0}\right\}} .
\end{aligned}
$$

Sea $T_{0}$ la descomposición de $T$ en $\left\{X_{1}=d_{0}\right\}$, dada por el lema 2.2. Tenemos entonces

$$
\begin{aligned}
E\left(\left(\frac{a+S_{T}}{m+T}\right) \mathbf{1}_{\left\{X_{1}=d_{0}\right\}}\right) & =E\left[E\left(\frac{a+d_{0}+S_{T_{0} \circ \theta}}{m+1+T_{0} \circ \theta} \mid \mathcal{F}_{1}\right) \mathbf{1}_{\left\{X_{1}=d_{0}\right\}}\right] \\
& =E\left(\frac{a+d_{0}+S_{T_{0}}}{m+1+T_{0}}\right)(1-p)
\end{aligned}
$$


(la última igualdad de la propiedad de Markov), y obtenemos

$$
E\left(W_{T}\right)=\frac{a+\mu}{m+1}+E\left(\frac{a+d_{0}+S_{T_{0}}}{m+1+T_{0}}-\frac{a+d_{0}}{m+1}\right)(1-p)
$$

Como

$$
E\left(\frac{a+d_{0}+S_{T_{0}}}{m+1+T_{0}}\right) \leq V_{N-1}\left(a+d_{0}, m+1\right)
$$

al reemplazar en (3) se obtiene

$$
E\left(W_{T}\right) \leq \frac{a+\mu}{m+1}+\bar{V}_{N-1}\left(a+d_{0}, m+1\right)(1-p)
$$

Esto demuestra que la cantidad

$$
g(d)=\frac{a+\mu}{m+1}+\bar{V}_{N-1}\left(a+d_{0}, m+1\right)(1-p),
$$

acota superiormente a $\sup _{T \in \mathcal{T}_{0}^{N}} E\left(W_{T}\right)$. Probaremos ahora que estas cantidades son iguales construyendo un $T \in \mathcal{T}_{0}^{N}$ tal que $E\left(W_{T}\right)=g(d)$. Para esto consideramos un $T_{0} \in \mathcal{T}^{N-1}$, tiempo de parada óptimo del juego con parámetros $a+d_{0}$ y $m+1$, esto es:

$$
E\left(W_{T_{0}}\right)=V_{N-1}\left(a+d_{0}, m+1\right) .
$$

Luego, por el teorema 1 existe $T \in \mathcal{T}_{0}^{N}$ tal que $\varphi_{N,\left\{d_{0}\right\}}(T)=T_{0}$, y entonces para este tiempo $T$ se obtiene de (3) que $E\left(W_{T}\right)=g(d)$, y consecuentemente

$$
\sup _{T \in \mathcal{T}_{0}^{N}} E\left(W_{T}\right)=\frac{a+\mu}{m+1}+\bar{V}_{N-1}\left(a+d_{0}, m+1\right)(1-p) .
$$

Similarmente se obtiene que

$$
\sup _{T \in \mathcal{T}_{1}^{N}} E\left(W_{T}\right)=\frac{a+\mu}{m+1}+\bar{V}_{N-1}\left(a+d_{0}, m+1\right)(1-p)+\bar{V}_{N-1}\left(a+d_{1}, m+1\right) p .
$$

Finalmente, de (1) obtenemos la primera identidad del teorema. La identidad (2) se obtiene de que $\bar{V}_{N-1}(a+x, m+1) \leq 0$ para $x$ en un intervalo de la forma $[c,+\infty[$.

\subsection{Caso de horizonte infinito}

El problema de cálculo (o de aproximación) de $V(a, m)$ se puede abordar a partir de los problemas de horizonte finito. En efecto, debido a la hipótesis sobre la distribución de los $X_{n}$, el proceso $W$ es de clase $\mathcal{D}$, y en virtud de los teoremas generales (ver por ejemplo la sección 3.7, capítulo 3 de [3]): $V(a, m)=\lim _{N \rightarrow \infty} V_{N}(a, m)$. Se deduce que podemos encontrar tiempos $\varepsilon$-óptimos del problema de horizonte infinito. También podemos deducir una relación recurrente para los $V(a, m)$. En efecto, de la relación recurrente (2) es lícito pasar al límite y obtener:

Teorema 4 En el caso de horizonte infinito se tiene:

$$
V(a, m)=\frac{a+\mu}{m+1}+E\left(\bar{V}\left(a+X_{1}, m+1\right)^{+}\right) .
$$




\section{Aplicaciones}

Las fórmulas del teorema 3 nos permiten establecer algunos resultados interesantes acerca de los valores del problema de parada óptima. Para esto interpretaremos los conjuntos de valores como sucesiones de funciones:

$$
V_{m}(\cdot)=V(\cdot, m), V_{m}^{N}(\cdot)=V_{N}(\cdot, m),
$$

y similarmente para los valores $\bar{V}(\cdot)$. Se define $h(a, m)=\frac{a+\mu}{m+1}-\frac{a}{m}$ si $m \geq 1$, y $h(a, 0)=$ $a+\mu$.

Por el teorema 3 se tienen las relaciones:

$$
\bar{V}_{m}^{N}(a)=h(a, m)+E\left(\bar{V}_{m+1}^{N-1}\left(a+X_{1}\right)^{+}\right), \bar{V}_{m}(a)=h(a, m)+E\left(\bar{V}_{m+1}\left(a+X_{1}\right)^{+}\right) .
$$

\subsection{Desigualdades fundamentales}

El siguiente resultado resume algunas de las propiedades más importantes de las sucesiones de valores:

Teorema 5 Para cada $a \in \mathbb{R}, m \in \mathbb{N}$ y $N \in \mathbb{N}$ se tiene

$$
\bar{V}_{m}(a) \geq h(a, m)+\left(\bar{V}_{m+1}(a+\mu)\right)^{+}, \quad \bar{V}_{m}^{N}(a) \geq h(a, m)+\left(\bar{V}_{m+1}^{N-1}(a+\mu)\right)^{+} .
$$

Equivalentemente:

$$
V_{m}(a) \geq \operatorname{máx}\left(\frac{a+\mu}{m+1}, V_{m+1}(a+\mu)\right), \quad V_{m}^{N}(a) \geq \operatorname{máx}\left(\frac{a+\mu}{m+1}, V_{m+1}^{N-1}(a+\mu)\right) .
$$

Prueba: Las primeras desigualdades son una consecuencia inmediata de la fórmula de recurrencia, y la desigualdad de Jensen. De la definición de $\bar{V}_{m}$ y $\bar{V}_{m}^{N}$ se obtienen las otras desigualdades.

El siguiente resultado nos proporciona cotas inferiores para los $\bar{V}$, que como veremos más adelante, son en cierta forma óptimas. Si bien estas desigualdades se pueden obtener en forma directa (tomando tiempos de parada constantes), es interesante verlas como corolario de las relaciones de recurrencia.

Corolario 4.1 Para $m \geq 0$ se tiene

$$
V_{m}(a) \geq \operatorname{máx}\left(\frac{a+\mu}{m+1}, \mu\right), \quad V_{m}^{N}(a) \geq \operatorname{máx}\left(\frac{a+\mu}{m+1}, \frac{a+N \mu}{m+N}\right) .
$$

Prueba: Basta observar que, por el teorema anterior:

$$
V_{m}^{N}(a) \geq V_{m+1}^{N-1}(a+\mu) \geq V_{m+2}^{N-2}(a+2 \mu) \ldots \geq V_{m+N-1}^{1}(a+(N-1) \mu)=\frac{a+N \mu}{m+N} .
$$

Esto demuestra la desigualdad en el caso de horizonte finito. Al tomar límites se obtiene el caso infinito.

El siguiente resultado será de utilidad posteriormente. 
Corolario 4.2 Si $N>1$ se tiene $V_{m}^{N}(m \mu)>\mu$, y equivalentemente: $\bar{V}_{m}^{N}(m \mu)>0$.

Prueba: La desigualdad $V_{m}^{N}(m \mu) \geq \mu$ se deriva directamente del corolario anterior. Para ver que la desigualdad es estricta, nótese que $d_{0}<\mu$, y por lo tanto $h\left(m \mu+d_{0}, m+1\right)>0$. Del corolario anterior tendremos entonces $\bar{V}_{m+1}^{N-1}\left(m \mu+d_{0}\right)>0$, y usando luego la relación recurrente tendremos:

$$
V_{m}^{N}(m \mu) \geq \mu+\bar{V}_{m+1}^{N-1}\left(m \mu+d_{0}\right)(1-p)>\mu .
$$

Nota: Dado que $V_{m}(m \mu) \geq V_{m}^{N}(m \mu)$, se obtiene también la desigualdad para horizonte infinito.

\subsection{Existencia de tiempos óptimos}

Es interesante que las cotas inferiores del corolario 4.1 realmente se alcanzan eventualmente, como lo demuestra el siguiente teorema.

Teorema 6 Para $a \geq(m+1) d_{1}-d_{0}$ se tiene

$$
\bar{V}_{m}^{N}(a)=\bar{V}_{m}(a)=h(a, m), \quad V_{m}(a)=V_{m}^{N}(a)=\frac{a+\mu}{m+1} .
$$

Si $N=1$ esto es válido para todo a. Si $N>1$, es necesario que a $>m \mu$. La relación (6) equivale a que el problema de parámetros a y $m$ (horizonte finito o infinito) admite el tiempo óptimo constante $T=1$.

Prueba: Sea $m$ un entero cualquiera. Por el lema 2.3 se deduce que la variable $\bar{V}_{m+1}\left(a+X_{1}\right)$ es negativa para $a \geq(m+1) d_{1}-d_{0}$, y en particular $E\left(\bar{V}_{m+1}^{+}\left(a+X_{1}\right)\right)=0$ para un tal $a$. De la relación recurrente deducimos la relación (6) para $V_{m}(a)$. Luego, como

$$
\frac{a+\mu}{m+1} \leq V_{m}^{N}(a) \leq V_{m}(a)
$$

se obtiene lo mismo para $V_{m}^{N}(a)$. La condición necesaria resulta del corolario 4.2.

Nota: El menor tal $a$ para el que se cumple $V_{m}^{N}(a)=\frac{a+\mu}{m+1}$, puede depender de $p$ y $N$, pero en todo caso se tiene $a \leq(m+1) d_{1}-d_{0}$, y $a>m \mu$.

Este resultado extiende los resultados de horizonte infinito sobre la existencia de tiempos de parada óptimos finitos, para problemas de parámetros $a=m=0$ (problema de medias aritméticas), bajo la hipótesis $\mu=0$ (ver [1]). En cierto sentido es más fuerte pues además de aseguar la existencia caracteriza el tiempo óptimo del problema, no supone que las variables sean centradas y trata valores arbitrarios de $a$ y $m$ (siendo $a$ suficientemente grande).

Corolario 4.3 Considere $m, N \in \mathbb{N}$ fijos. Entonces las funciones $V_{m}$ y $V_{m}^{N}$ son contractivas en todo $\mathbb{R}$, con constante de contracción $1 /(m+1)$. Esta constante es óptima.. 
Prueba: Basta demostrar el resultado para las $V_{m}$. Debido a la convexidad, para $a<b$ el cociente

$$
\frac{V_{m}(b)-V_{m}(a)}{b-a}
$$

crece conforme $a$ y $b$ aumentan (manteniendo fijo $a-b$ ). Por el teorema anterior, el cociente coincide com $\frac{1}{m+1}$ para $a$ y $b$ suficientemente grandes. Luego se tiene

$$
\frac{V_{m}(b)-V_{m}(a)}{b-a} \leq \frac{1}{m+1}
$$

\subsection{Variaciones según el parámetro}

En horizonte finito, ciertas propiedades de variación de $V_{m}^{N}(a)$, como función de los parámetros $m, N, a$, se pueden deducir fácilmente del hecho que $V_{m}^{N}(\cdot)$ es la envolvente superior de una familia finita de rectas. Por ejemplo, no es difícil ver que $V_{m}^{N}(\cdot)$ es estrictamente creciente, convexa, y que además es afín lejos del origen. A continuación estudiaremos algunos resultados análogos en el caso de horizonte infinito, así como la variación de $V_{m}^{N}(a)$ en función de $N$.

Denotemos por $c_{m}$ el único cero de $\bar{V}_{m}$. Los resultadosde las secciones anteriores, pueden ser traducidos en términos de ciertas propiedades de la sucesión $\left(c_{m}\right)$.

Lema 4.1 La sucesión $\left(c_{m}\right)_{m}$ cumple las siguientes propiedades:

1. Para cada $m$ se tiene $m \mu<c_{m} \leq m d_{1}$.

2. Si $m \geq 1$ se tiene $c_{m}<c_{m+1}-d_{0}$.

3. La relación $\bar{V}_{m}(a)=h(a, m)$ se cumple sii $a \geq c_{m+1}-d_{0}$.

En efecto, la propiedad 1 es la traducción del corolario 4.2 y el lema 2.3. Luego, si $a \geq c_{m+1}-d_{0}$ se sigue que $\bar{V}(a+x, m+1) \leq 0$ para todo $x \in D$, de donde por la relación recurrente se concluye que

$$
\bar{V}(a, m)=\frac{m \mu-a}{m(m+1)} \leq 0
$$

dado que $a \geq c_{m+1}+d_{0} \geq(m+1) \mu-d_{0} \geq m \mu$. Esto demuestra la propiedad 2. La propiedad 3 se deduce también de la propiedad 1 y la relación recurrente.

\subsubsection{Variación según $a$}

Como se ha dicho, en el problema de horizonte finito es fácil ver que para todo $m \geq 0$ la función $V_{m}^{N}$ es estrictamente creciente. Es natural plantearse en horizonte infinito cómo son los intervalos de crecimiento estricto de las funciones $V_{m}, m \geq 0$.

Siendo cada $V_{m}$ creciente y convexa, la función derivada a la derecha de $V_{m}$, que denotamos $D^{+} V_{m}$, existe en todo punto y es creciente y no negativa. Entonces el intervalo:

$$
I_{m}=\left\{a: D^{+} V_{m}(a)>0\right\}
$$


es un intervalo abierto en $+\infty$, y no es vacío pues $V_{m}$ es función afín no constante en un intervalo abierto en $+\infty$. Está claro que $I_{m}$ es el intervalo máximo de crecimiento estricto de $V_{m}$, ya que su complemento es un subintervalo abierto en $-\infty$ donde $D^{+} V_{m}=0$. La propiedad de crecimiento estricto de las $V_{m}$ está pues determinada por el signo de $D^{+} V_{m}$. Nótese que $D^{+} \bar{V}_{m}=D^{+} V_{m}+t_{m}$, donde $t_{m}=-\frac{1}{m}$ si $m>0$, y $t_{0}=0$. Así pues

$$
I_{m}=\left\{a: D^{+} \bar{V}_{m}(a)>t_{m}\right\}
$$

Definimos $H(0)=1 \mathrm{y}$

$$
H(m)=\frac{-1}{m(m+1)}, m \neq 0 .
$$

Por $X$ se denota una variable de ley $G$. Como consecuencia del teorema 4 se tiene:

Lema $4.2 D^{+} \bar{V}_{m}(a)=H(m)+E\left(D^{+} \bar{V}_{m+1}^{+}(a+X)\right)$.

Para $m \geq 1$, consideremos el intervalo $C_{m}=\left[c_{m},+\infty\right.$ [, que según los preliminares es no vacío y distinto de $\mathbb{R}$.

Teorema 7 Para $m \geq 0$ se cumple: $I_{m+1} \bigcup C_{m+1}=I_{m}+d_{1}$

Prueba: Gracias a la relación recurrente del lema anterior, y al hecho que $t_{m+1}+H(m)=$ $t_{m}$, se deduce que la relación $a \in I_{m}$ es equivalente a la desigualdad

$$
E\left(D^{+} \bar{V}_{m+1}^{+}(a+X)\right)>t_{m+1}
$$

Pero dado que $D^{+} \bar{V}_{m+1}^{+}(a+X) \geq t_{m+1}$, y siendo la función $D^{+} \bar{V}_{m+1}^{+}$creciente, la desigualdad (7) se cumple si y solo si se cumple

$$
D^{+} \bar{V}_{m+1}^{+}\left(a+d_{1}\right)>t_{m+1}
$$

Como $t_{m+1}<0$, esta condición se cumple para $a+d_{1} \in C_{m+1}$, demostrando que $C_{m+1} \subseteq$ $I_{m}+d_{1}$. Por otro lado, dado que $D^{+} \bar{V}_{m+1}^{+}$y $D^{+} \bar{V}_{m+1}$ coinciden fuera de $C_{m+1}$, para $a+d_{1} \in\left(I_{m}+d_{1}\right)-C_{m+1}$ la condición (8) equivale a $a+d_{1} \in I_{m+1}$, lo que prueba la identidad buscada.

Corolario 4.4 Si para algún $m_{0} \geq 0$ se cumple $I_{m_{0}}=\mathbb{R}$, entonces $I_{m}=\mathbb{R}$ para todo $m \in \mathbb{N}$.

Prueba: Puesto que $C_{m} \neq \mathbb{R}$ para $m \geq 1$, la relación del teorema anterior muestra claramente que $I_{m}=\mathbb{R}$ sii $I_{m+1}=\mathbb{R}$. Por lo tanto $I_{m}=\mathbb{R}$ para todo $m \in \mathbb{N}$ si se cumple al menos para algún $m$. 


\subsubsection{Variación según $m$}

En el caso de horizonte finito, es evidente que

$$
\lim _{m \rightarrow \infty} V_{m}^{N}(a)=\lim _{m \rightarrow \infty} \bar{V}_{m}^{N}(a)=0 .
$$

El caso de horizonte infinito es tratado a continuación. Se definen las funciones $f$ y $g$ así:

$$
f(a)=\overline{\lim } V_{m}(a)=\overline{\lim } \bar{V}_{m}(a), \quad g(a)=\underline{\lim } V_{m}(a)=\underline{\lim } \bar{V}_{m}(a) .
$$

El siguiente teorema demuestra en particular que $f$ y $g$ son finitas.

Teorema 8 La sucesión $\left(\bar{V}_{m}(m \mu)\right)_{m \in \mathbb{N}}$ es convergente, decreciente y de límite no negativo. La sucesión $\left(V_{m}(m \mu)\right)_{m \in \mathbb{N}}$ es convergente, decreciente y de límite no menor que $\mu$. Las sucesiones $\left(V_{m}(a)\right)_{m \in \mathbb{N}} y\left(\bar{V}_{m}(a)\right)_{m \in \mathbb{N}}$ son acotadas para cualquier a, y las funciones $f$ y $g$ son constantes y positivas.

Prueba: Por el teorema 5 tenemos que

$$
\bar{V}_{m}(m \mu) \geq\left(\bar{V}_{m+1}((m+1) \mu)\right)^{+} \geq \bar{V}_{m+1}((m+1) \mu),
$$

de donde se obtiene que $\left(\bar{V}_{m}(m \mu)\right)$ es no negativa y decreciente. Esto demuestra la primera afirmación. Como $V_{m}(m \mu)=\bar{V}_{m}(m \mu)+\mu$ se obtiene lo mismo para $\left(V_{m}(m \mu)\right)$. Luego, por el corolario 4.3 tenemos que

$$
\left|\bar{V}_{m}(a)-\bar{V}_{m}(m \mu)\right| \leq \frac{|a-m \mu|}{m+1} \rightarrow|\mu|,
$$

y como $\left(\bar{V}_{m}(m \mu)\right)$ converge, obtenemos la acotación de $\left(\bar{V}_{m}(a)\right)$, y por ende la de $\left(V_{m}(a)\right)$. Finalmente, como $\bar{V}_{m}(a)$ es decreciente y $V_{m}(a)$ creciente en $a$, se obtiene inmediatamente que $f$ y $g$ son crecientes y decrecientes, y por lo tanto constantes.

Teorema 9 Supongamos que $\mu \geq 0$. Entonces:

1. Para todo a $\in \mathbb{R}$ la sucesión $\left(V_{m}(a)\right)_{m \in \mathbb{N}}$ es decreciente.

2. Para $a \leq 0$ la sucesión $\left(\bar{V}_{m}(a)\right)_{m \geq 1}$ es decreciente, estrictamente si $a<0$.

3. Las sucesiones de funciones $\left(V_{m}\right)_{m \in \mathbb{N}} y\left(\bar{V}_{m}\right)_{m \in \mathbb{N}}$ convergen a una función constante $c \geq \mu$, uniformemente en cada compacto de $\mathbb{R}$.

Prueba: Por el teorema 5 tenemos

$$
V_{m}(a) \geq V_{m+1}(a+\mu) \geq V_{m+1}(a),
$$

la segunda desigualdad por ser $V_{m}$ creciente en $a$. Por el terorema de Weierstrass $\left(V_{m}(a)\right)_{m \in \mathbb{N}}$ es convergente, y por el corolario 4.1 se tiene $c=\operatorname{lím} V_{m}(0) \geq \mu$. Luego

$$
\left|V_{m}(a)-c\right| \leq\left|V_{m}(a)-V_{m}(0)\right|+\left|V_{m}(0)-c\right| \leq \frac{a}{m+1}+\left|V_{m}(0)-c\right|
$$

de donde se obtiene inmediatamente que $\left(V_{m}\right)$ converge a $c$, uniformemente en compactos. El resto se obtiene inmediatamente de la definición de $\bar{V}_{m}(a)$.

Corolario 4.5 En el caso $\mu \geq 0$ se tiene $f(a)=g(a)=\lim _{m \rightarrow \infty} V_{m}(0)$. 


\subsubsection{Variación según $N$}

La sucesión $\left(V_{N}(a, m)\right)_{N \geq 1}$ de los valores de los juegos de horizonte finito es, para cualesquiera valores de los parámetros, una sucesión creciente de reales. Es natural preguntarse si tal sucesión es estrictamente creciente. No siempre es así, como lo muestra el ejemplo con $D=\{-1,1\}$, pues en este caso $V_{1}(1,1)=\frac{1}{2}=V_{2}(1,1)$. Más aún, por el teorema 6 se tiene que $V_{N}(a, m)$ es constante en $N$ para $a \geq m+2$, y por el siguiente resultado, esto es válido si $a \geq 1$.

Teorema 10 Son equivalentes:

(i) La sucesión $\left(V_{N}(a, m)\right)_{N \geq 1}$ es estrictamente creciente.

(ii) $V_{1}(a, m)<V_{2}(a, m)$.

(iii) $a<m \mu+\left(\mu-d_{0}\right)$.

Prueba: Evidentemente (i) implica (ii). Por otro lado, por el teorema 3 tenemos que la condición $V_{1}(a, m)<V_{2}(a, m)$ es equivalente a

$$
\int\left(\bar{V}_{1}(a+x, m+1)\right)^{+} d G(x)>0,
$$

y como

$$
\bar{V}_{1}(a+x, m+1)=\frac{(m+1) \mu-a-x}{(m+1)(m+2)}
$$

se sigue que lo anterior se cumple si y solo si $x<(m+1) \mu-a$ para algún $x \in D$, lo cual equivale a tener $d_{0}<(m+1) \mu-a$. Esto demuestra la equivalencia entre (ii) y (iii).

Basta entonces con demostrar que (iii) implica (i). Para esto consideremos el siguiente conjunto $S=\left\{N \in \mathbb{N}:\left[a<m \mu+\left(\mu-d_{0}\right) \Rightarrow V_{N}(a, m)<V_{N+1}(a, m)\right]\right\}$.

Como (iii) implica (ii) tenemos que $1 \in S$. Supongamos ahora que $N-1 \in S$, y tomemos $a, m$ tales que $a<m \mu+\left(\mu-d_{0}\right)$. Como $d_{0} \leq \mu$ tenemos que $a+d_{0}<(m+1) \mu+\left(\mu-d_{0}\right)$, y como $N-1 \in S$ se concluye que $V_{N-1}\left(a+d_{0}, m+1\right)<V_{N}\left(a+d_{0}, m+1\right)$..

Por otro lado,

$$
V_{N-1}\left(a+d_{0}, m+1\right) \geq V_{1}\left(a+d_{0}, m+1\right)=\frac{a+d_{0}+\mu}{m+2}>\frac{a+d_{0}}{m+1}
$$

(pues $\left.a+d_{0}<(m+1) \mu\right)$, y por lo tanto

$$
0<\bar{V}_{N-1}\left(a+d_{0}, m+1\right)<\bar{V}_{N}\left(a+d_{0}, m+1\right) .
$$

Tenemos entonces

$$
\left(\bar{V}_{N-1}\left(a+d_{0}, m+1\right)\right)^{+}<\left(\bar{V}_{N}\left(a+d_{0}, m+1\right)\right)^{+},
$$

y como la desigualdad no estricta es válida en todo $D$ obtenemos

$$
\int\left(\bar{V}_{N-1}(a+x, m+1)\right)^{+} d G(x)<\int\left(\bar{V}_{N}(a+x, m+1)\right)^{+} d G(x) .
$$


Esto implica $V_{N}(a, m)<V_{N+1}(a, m)$, con lo cual $N \in S$. El principio de inducción se encarga del resto.

Note que en particular

$$
V_{1}(a, m)=V_{2}(a, m) \Leftrightarrow a \geq m \mu+\left(\mu-d_{0}\right),
$$

pero el resultado no dice qué pasa con $\left(V_{N}\right)_{N \geq 2}$. El siguiente resultado resuelve parcialmente este asunto. Su demostración se sigue por inducción.

Lema 4.3 Para $k \in \mathbb{N}$ y $a \geq m \mu+k\left(\mu-d_{0}\right)$ se tiene

$$
V_{1}(a, m)=V_{2}(a, m)=\ldots=V_{k+1}(a, m)=\frac{a+\mu}{m+1} .
$$

\section{Cotas inferiores en el caso $d_{0} \geq 0$}

No es en principio fácil resolver (5). Sin embargo, puede ser útil para deducir propiedades generales de los $V(a, m)$, de la misma manera como se hizo en horizonte finito para estudiar la estructura de los $V_{N}(a, m)$.

Como ejemplo de aplicación, consideremos el caso particular $D=\{0,1\}$. Buscamos una cota inferior para $V(0, m)$, con $m \in \mathbb{N}$. En este caso la relación (5) se convierte en

$$
V(0, m)=\operatorname{máx}\left(\frac{p}{m+1}+(1-p) V(0, m+1),(1-p) V(0, m+1)+p V(1, m+1)\right),
$$

dado que $V(0, m+1) \geq 0$. En particular:

$$
V(0, m) \geq \frac{p}{m+1}+(1-p) V(0, m+1) .
$$

Iterando esta desigualdad se obtiene:

$$
V(0, m) \geq p\left[\frac{1}{m+1}+\frac{1-p}{m+2}+\cdots+\frac{(1-p)^{k}}{m+k+1}\right]+(1-p)^{k+1} V(0, m+k+1) .
$$

Notemos ahora que en este caso particular se tiene $0 \leq V(0, m) \leq 1$, para todo $m \in \mathbb{N}$, dado que

$$
0 \leq W_{T}=\frac{S_{T}}{m+T} \leq \frac{T}{m+T} \leq 1, \forall T \in \mathcal{T}
$$

Por lo tanto tenemos que $(1-p)^{k+1} V(0, m+k+1) \rightarrow 0$, cuando $k \rightarrow \infty$. Tomando el límite en la desigualdad (9) obtenemos

$$
V(0, m) \geq p \sum_{k=1}^{\infty} \frac{1}{m+k}(1-p)^{k-1}
$$

En el caso particular que $m=0$ se tiene:

$$
V(0,0) \geq p \sum_{k=1}^{\infty} \frac{1}{k}(1-p)^{k-1}=\frac{p}{1-p} \sum_{k=1}^{\infty} \frac{(1-p)^{k}}{k}=\frac{-p \ln p}{1-p} .
$$


En [1] se obtiene $V(0,0) \geq p(2-p)$. Nuestra cota es mejor para $p$ en el intervalo $] 0, p_{0}[$, donde $p_{0}$ es la primera raíz positiva de $p(2-p)=\frac{-p \ln p}{1-p}$. Aproximadamente se tiene $p_{0}=0,316197376 \ldots$

Para $m=1$, un cálculo similar nos lleva a $V(0,1) \geq-\frac{p \ln p}{(1-p)^{2}}-\frac{p}{1-p}$. Esta cota se puede comparar con la cota $p\left(\frac{3}{2}-p\right)$, obtenida de considerar el tiempo de parada

$$
T=\left\{\begin{array}{cc}
1 & \text { si } X_{1}=1 \\
\inf \left\{n: \frac{S_{n}}{n+1}>p-\varepsilon\right\} & \text { si } X_{1} \neq 1,
\end{array}\right.
$$

y luego hacer $\varepsilon$ tender a cero. Al igual que en el caso $m=0$, la cota logarítmica es mejor para valores pequeños de $p$.

Generalización: Analicemos ahora el caso general, con $W_{n}=\frac{a+S_{n}}{m+n}$, con la única restricción que $d_{0} \geq 0$. En este caso se tiene

$$
\begin{aligned}
V(a, m) & \geq \frac{p\left(a+d_{1}\right)}{m+1}+(1-p) V\left(a+d_{0}, m+1\right) \\
& \geq \frac{p\left(a+d_{1}\right)}{m+1}+(1-p) V(a, m+1),
\end{aligned}
$$

pues $a+d_{0} \geq a$. Iterando se obtiene

$$
\begin{aligned}
V(a, m) & \geq p\left(a+d_{1}\right) \sum_{k=1}^{\infty} \frac{1}{m+k}(1-p)^{k-1} \\
& =\frac{p\left(a+d_{1}\right)}{(1-p)^{m+1}}\left(-\ln p-P_{m}(1-p)\right)
\end{aligned}
$$

donde $P_{m}(x)=\sum_{k=1}^{m} \frac{x^{k}}{k}$

\section{Algoritmos para el caso de horizonte finito}

La fórmula de recurrencia (2) nos proporciona un algoritmo eficaz para el cálculo del valor del juego en el caso de horizonte infinito. En efecto, para calcular $V_{N}(a, m)$ se deben calcular primero los $V_{N-1}(a+d, m+1)$, para $d \in D$. Pero para calcular estos valores se deben calcular antes los valores $V_{N-2}\left(a+d+d^{\prime}, m+2\right)$, para $d, d^{\prime} \in D$. Esto es, se deben calcular los $V_{N-2}(a+x, m+2)$, para $x \in D^{(2)}$, donde

$$
D^{(2)}=D+D=\left\{2 d_{0}, d_{0}+d_{1}, 2 d_{1}\right\}
$$

Por inducción definimos $D^{(n+1)}=D^{(n)}+D$, y resulta que el algoritmo requiere calcular $\operatorname{los} V_{k}(a+x, m+N-k)$ para $k=1,2, \ldots, N-1$ y $x \in D^{(N-k)}$. Nótese que

$$
D^{(n)}=\left\{n d_{0},(n-1) d_{0}+d_{1}, \ldots, d_{0}+(n-1) d_{1}, n d_{1}\right\}
$$

tiene cardinalidad $n+1$. Con este algoritmo no es difícil crear un programa que calcule los valores del juego, así como el tiempo óptimo. 
Ejemplo 6.1 En el caso $D=\{-1,1\}$ y $p=, 5$, la siguiente tabla incluye algunos valores aproximados. Esta refleja propiedades interesantes del valor del juego, algunas de las cuales ya hemos demostrado.

\begin{tabular}{c|ccccccc}
\hline \hline$m$ & $V_{m}^{100}(-7)$ & $V_{m}^{100}(-4)$ & $V_{m}^{100}(-1)$ & $V_{m}^{100}(0)$ & $V_{m}^{100}(1)$ & $V_{m}^{100}(2)$ & $V_{m}^{100}(3)$ \\
\hline 0 &,- 056004 &, 000696 &, 186051 &, 567788 & 1,152973 & 2 & 3 \\
3 &,- 055800 &,- 006248 &, 097168 &, 175686 &, 325370 &, 508031 &, 75 \\
10 &,- 054334 &,- 013572 &, 050252 &, 083836 &, 130069 &, 196827 & .27 \\
100 &,- 033285 &,- 016469 &, 001986 &, 008704 &, 015809 &, 023381 &, 031520 \\
1000 &,- 006320 &,- 003552 &,- 000754 &, 000188 &, 001136 &, 002091 &, 003054 \\
\hline \hline
\end{tabular}

Para $N=20$ y $a=m=0$, el tiempo óptimo está definido por el vector:

$$
c=[1,1,1,1,1,2,2,2,2,2,2,1,1,1,1,1,1,1,0,-20] .
$$

\section{Referencias}

[1] Chow, Y.S., Robbings, H.; Siegmund, D. (1991) The Theory of Optimal Stopping. Dover Pub. Inc., New York.

[2] Chow, Y.S.; Robbings, H. (1965) "On optimal stopping rules for $S_{n} / n$ ", Illinois Journal of Mathematics.

[3] Dalang, R.C.; Cairoli, R. (1996) Sequential Stochastic Optimization. John Wiley \& Sons, Inc., New York.

[4] Dellacherie; Meyer (1978) Probabilité et Potentiel. Hermann, Paris.

[5] Lobo, J.; Cambronero, S. (2001) "Problemas de parada óptima para promedios generalizados de variables discretas. Parte I: Fórmulas de recurrencia". Preprint, CIMPA11-2001.

[6] Maitra, A.P.; Sudderth, W.D. (1996) "Discrete gambling and stochastic games", Appl. of Math., Springer-Verlag, New York.

[7] Roberts, A.W.; Varberg, D.E. (1973) Convex Functions. Academic Press, New York. 